\title{
New Immunological Technique for Diagnosis of Candida albicans Infection
}

\section{Russul Arkan Hassan}

An M Sc thesis conducted in Baghdad University, College of Education for Pure Sciences (Ibn Al-Haitham), 2019, under the supervision of Assistant Professor Dr. Hazima Mossa Al-Abassi

Background: Candida albicans infections that involve skin and mucous membrane is the most common opportunistic fungal infection. Although, the infection is mild and superficial, however, it is with psychological burden especially in women. Additionally, the infection may be serious and difficult to control in immunosuppressed individuals such as those with diabetes. Early and specific diagnosis is essential to achieve effective treatment outcomes.

Aim: To develop a new technique for diagnosis of Candida albicans (skin, mouth, and vagina) infection.

Methodology: This study included 100 samples of the patient (immunocompromised women with average age (55-57) year and babies with average age (1-18 month), patients infected with various (fungi, bacteria) infection, in addition to 20 samples for an apparently healthy individual (control). Samples were collected from ALDowaly Private Hospital in Baghdad during the period January 2019 to June 2019. All infections were diagnosed by consultant medical staff at the hospital by using several tests including (germ tube, culture, Vitek and API20C). Cases enrolled in this study were as follow:

- Thirty- Samples from patients with C. albicans infection (skin scales).

- Fifteen-Samples from patients with Aspergillus spp. infection (skin scales).

- Fifteen-Samples from patients with Trichophyton spp. infection (skin scales). The skin scales were collected from intertriginious area.

- Ten-Samples from patients with C. albicans infection (skin swabs). 
- Ten-Samples from patient's bacteria with Staphylococcus aureus infection (skin swabs).

- Ten-Samples from patients (1-18 month) infected with $C$. albicans (mouth swabs).

- Ten-Samples from patients with C. albicans infection (vaginal swabs).

Results: The samples were examined using the new technique compared to the routine methods of diagnosis. The new technique gave a positive result for samples infected with $C$. albicans, while the none Candida albicans infection (fungal, bacteria) gave a negative result. The Vitek test was with high sensitivity (100\%) and specificity (100\%) for the diagnosis of C. albicans than other methods such as API20C [sensitivity (100\%) and specificity (97\%)], Colonial morphology "culture" [sensitivity (96\%) and specificity (93\%)] and germ tube test [sensitivity (98\%) and specificity (95\%)]. The results of the new technique test in this study for the diagnosis of $C$. albicans was similar to the result of Vitek test in sensitivity $(100 \%)$ and specificity $(100 \%)$. The benefits of this new technique are to provide information with high accuracy, cost- effective (low-cost) and reduced time consumption. For example, physicians can perform it through the attending visit of the patient without having to be sent to the laboratory.

Conclusion: The new technique was with high sensitivity and specificity in diagnosis of Candida albicans infection. 